Check for updates

Cite this: Phys. Chem. Chem. Phys., 2019, 21, 18999

Received 3rd June 2019

Accepted 6th August 2019

DOI: $10.1039 / c 9 c p 03127 \mathrm{~h}$

rsc.li/pccp

\section{Multistate hybrid time-dependent density functional theory with surface hopping accurately captures ultrafast thymine photodeactivation}

\begin{abstract}
Shane M. Parker, (D) $* \dagger$ Saswata Roy (D) and Filipp Furche (D)*
We report an efficient analytical implementation of first-order nonadiabatic derivative couplings between arbitrary Born-Oppenheimer states in the hybrid time-dependent density functional theory (TDDFT) framework using atom-centered basis functions. Our scheme is based on quadratic response theory and includes orbital relaxation terms neglected in previous approaches. Simultaneous computation of multiple derivative couplings and energy gradients enables efficient multistate nonadiabatic molecular dynamics simulations in conjunction with Tully's fewest switches surface hopping (SH) method. We benchmark the thus obtained multistate TDDFT-SH scheme by simulating ultrafast decay of UV-photoexcited thymine, for which accurate gas-phase data from ultrafast spectroscopy experiments are available. The calculations predict a fast $153 \mathrm{fs}$ decay from the bright $\mathrm{S}_{2}$ to the dark $\mathrm{S}_{1}$ excited state, followed by a much slower 14 ps $S_{1}$ deactivation to the ground state; statistical uncertainties were estimated using bootstrap sampling. These results agree well with the experimentally observed time constants of 100-200 fs and 5-7 ps, respectively, unlike previous multiconfigurational self-consistent field and second-order algebraic diagrammatic construction calculations. Furthermore, our results support the $\mathrm{S}_{1}$-trapping hypothesis [J. J. Szymczak et al., J. Phys. Chem. A, 2009, 113, 12686-12693]. For thymine, the computational cost of a single TDDFT-SH time-step including the lowest 3 states, all couplings and gradients, is $\sim 5$ times larger than the cost of a single Born-Oppenheimer dynamics time step for the ground state in our implementation. Thus, ps nonadiabatic dynamics simulations using multistate hybrid TDDFT-SH for systems with up to $\sim 100$ atoms are possible without drastic approximations on single workstation nodes. Our implementation will be made available through Turbomole.
\end{abstract}

\section{Introduction}

Nonadiabatic molecular dynamics ${ }^{1}$ (NAMD) using a combination of time-dependent density functional theory ${ }^{2-7}$ (TDDFT) and surface hopping $^{8}(\mathrm{SH})$ has emerged as a versatile tool for studying and analyzing complex photochemical transformations. ${ }^{9-11}$ TDDFT-SH has proven capable of predicting the kinetics of pericyclic ringopening and -closing reactions, ${ }^{12-20}$ the reactivity of photoexcited metal oxides ${ }^{21}$ such as perovskites ${ }^{22}$ and titania, ${ }^{23-25}$ and the branching ratios, kinetic energy distributions, and mass distributions of photodissociation reactions. ${ }^{26,27}$

However, the majority of photochemical TDDFT-SH applications to date have included only the ground state and the first excited state. Simulations including several excited states and all nonadiabatic couplings between them have been reported,

Department of Chemistry, University of California, Irvine, 1102 Natural Sciences II, Irvine, CA 92697-2025, USA. E-mail: filipp.furche@uci.edu, smparker@uci.edu $\dagger$ Present address: Department of Chemistry, Case Western Reserve University, 10900 Euclid Ave., Cleveland, OH 44106, USA. but rely on approximations with questionable validity such as the neglect of orbital relaxation ${ }^{17,19,28-32}$ or single Slater determinant models for the excited state. A rigorous theoretical approach to general couplings between excited states in the TDDFT framework has only recently been devised, ${ }^{33-35}$ but little is known about its performance in photochemical applications.

Here we report an efficient analytical implementation of state-to-state nonadiabatic couplings and multistate TDDFT-SH dynamics, which builds on these theoretical results and the implementation of the TDDFT quadratic response properties reported by our group previously. ${ }^{36}$ All required gradients and couplings can be computed simultaneously; as a result, the computational cost for a multistate NAMD time-step is proportional to that of a ground-state Born-Oppenheimer dynamics step, with a proportionality constant growing sublinearly with the number of electronic states.

We assess the performance of multistate TDDFT by investigating excited state decay of UV-photoexcited thymine. The experimental lifetime of UV-photexcited thymine, 5-7 ps, is significantly longer than that of other photoexcited nucleobases, ${ }^{37}$ and 
its decay has been extensively studied experimentally ${ }^{37-44}$ and computationally. ${ }^{43,45-53}$ Nevertheless, the deactivation mechanism of UV-photoexcited thymine remains controversial. ${ }^{37,45,53}$ In particular, prior NAMD-SH simulations using multiconfiguration selfconsistent field (MCSCF) and the second-order algebraic diagrammatic construction $[\mathrm{ADC}(2)]$ methods lead to excited-state lifetimes hard to reconcile with each other and with ultrafast pump-probe experiments. $^{37,43,50,52}$

We summarize the working equations and details of our implementation in Sections 2 and 3, followed by the results for thymine excited-state deactivation in Section 4 and conclusions in Section 5.

\section{Nuclear dynamics: fewest switches surface hopping}

Mixed quantum-classical methods treat nuclei classically and expand an auxiliary electronic wavefunction in a few-state (often adiabatic) electronic basis parametrically dependent on the nuclear coordinates. ${ }^{8}$ In our implementation, the auxiliary electronic density matrix

$$
\hat{\sigma}^{\mathrm{aux}}=\sum_{n m} \sigma_{n m}^{\mathrm{aux}}(t)\left|\phi_{n} ; \mathbf{R}(t)\right\rangle\left\langle\phi_{m} ; \mathbf{R}(t)\right|,
$$

is the basic dynamic variable, where $\mathbf{R}(t)$ denotes the nuclear coordinates, $\sigma_{n m}^{\text {aux }}(t)$ are expansion coefficients at time $t$, and $\phi_{n}$ are electronic basis states. ${ }^{8,54,55}$ The auxiliary electronic density matrix evolves as

$$
\dot{\sigma}^{\mathrm{aux}}(t)=-i\left[\overline{\mathbf{H}}, \sigma^{\mathrm{aux}}(t)\right]
$$

where $\overline{\mathbf{H}}=\mathbf{H}-i \mathbf{W}$ is the effective Hamiltonian, $H_{n m}=$ $\left\langle\phi_{n} ; \mathbf{R}(t)|\hat{H}| \phi_{m} ; \mathbf{R}(t)\right\rangle$ is a matrix element of the electronic Hamiltonian,

$$
W_{n m}=\left\langle\phi_{n} ; \mathbf{R}(t) \mid \frac{\partial}{\partial t} \phi_{m} ; \mathbf{R}(t)\right\rangle=\tau_{n m} \cdot \dot{\mathbf{R}}
$$

is a nonadiabatic coupling matrix element and

$$
\tau_{n m}^{(\xi)}=\left\langle\phi_{n} ; \mathbf{R}(t) \mid \frac{\partial}{\partial \xi} \phi_{m} ; \mathbf{R}(t)\right\rangle
$$

is the first-order derivative coupling between electronic states $n$ and $m .^{8}$

Within surface hopping, the electronic properties for a given trajectory at any give time are determined by a single BornOppenheimer electronic state, here referred to as the active state or active surface. ${ }^{54}$ This picture implicitly defines an active electronic density matrix,

$$
\sigma_{n m}^{\text {active }}=\delta_{n k} \delta_{m k}
$$

where $k$ labels the active electronic state. Transitions between electronic states are mimicked through stochastic hops of the active state.

FSSH is a specific realization of the surface hopping framework in which the probability of hopping from active state $k$ to electronic state $n$ in the time interval $t$ to $t^{\prime}$,

$$
\begin{aligned}
g_{k \rightarrow n}\left(t, t^{\prime}\right)= & \int_{t}^{t^{\prime}} \mathrm{d} T \\
& \times\left(\sigma_{n k}^{\mathrm{aux}}(T) \bar{H}_{k n}(T)-\bar{H}_{n k}(T) \sigma_{k n}^{\mathrm{aux}}(T)\right) / \sigma_{k k}^{\mathrm{aux}}(T)
\end{aligned}
$$

is chosen to reproduce the swarm average population of state $k$-that is, so $N_{k}(t) / N_{\text {traj }} \approx \sigma_{k k}(t)$ where $N_{k}(t)$ is the number of trajectories on active surface $k$ and $N_{\text {traj }}$ is the total number of trajectories. $^{8}$ In practice, surface hops are decided by computing hopping probabilities according to eqn (6) and generating a uniform random number $\eta \in[0,1]$. When $\eta<g_{k \rightarrow n}$, a surface hop to state $n$ is initiated and the momentum along the derivative coupling vector is rescaled to conserve energy. ${ }^{8}$ In case of insufficient kinetic energy, i.e. a frustrated hop, the hop is rejected and no momentum readjustment is performed.

\section{Electronic dynamics: TDDFT}

The NAMD frameworks described above require as input the adiabatic energies of electronic states $\left(E_{n}\right)$, nuclear forces $\left(\nabla E_{n}\right)$, and derivative couplings $\left(\tau_{n m}^{(\xi)} \equiv\left\langle\phi_{n} \mid \nabla_{\xi} \phi_{m}\right\rangle\right)$. All of these quantities are computed using (TD)DFT: ground state properties are determined from ground state DFT, excitation energies and derivative couplings between the ground state and an excited electronic state (ground-to-excited-state) are determined from linear response, and excited-state forces and derivative couplings between two excited electronic states (state-to-state) are determined from the quadratic response function.

\subsection{Linear response}

In the TDDFT linear response framework, ${ }^{2-4,56,57}$ excitation energies are obtained by solving the eigenvalue problem

$$
\left(\Lambda-\Omega_{n} \Delta\right)\left|X^{n}, Y^{n}\right\rangle=0,
$$

where

$$
\Lambda=\left(\begin{array}{cc}
\mathbf{A} & \mathbf{B} \\
\mathbf{B}^{*} & \mathbf{A}^{*}
\end{array}\right)
$$

is the linear response operator;

$$
\begin{aligned}
(A+B)_{i a, j b}= & \varepsilon_{a b} \delta_{i j}-\varepsilon_{i j} \delta_{a b}+2 f_{i a, j b}^{\mathrm{xc}}+2(i a \mid j b) \\
& -c_{x}[(i b \mid j a)+(i j \mid a b)] \\
(A-B)_{i a, j b}= & \varepsilon_{a b} \delta_{i j}-\varepsilon_{i j} \delta_{a b}+c_{x}[(i b \mid j a)-(i j \mid a b)],
\end{aligned}
$$

denote the electronic and magnetic orbital rotation Hessians, respectively;

$$
\Delta=\left(\begin{array}{cc}
1 & 0 \\
0 & -1
\end{array}\right)
$$

is the pseudometric, and $\Omega_{n}$ and $\left|X^{n}, Y^{n}\right\rangle$ are the eigenvalue (excitation energy) and associated eigenvector of excited state $n$, respectively. ${ }^{4,56}$ Here, indices $i, j, \ldots$ denote occupied, $a, b, \ldots$ 
virtual, and $p, q, \ldots$ general Kohn-Sham molecular orbitals. Moreover, $\varepsilon_{p q}$ is an element of the Kohn-Sham matrix which is assumed to be block-diagonal (i.e., the occupied-virtual blocks vanish), $f_{p q, r s}^{\mathrm{xc}}$ is an element of the exchange-correlation kernel, $(p q \mid r s)$ is an element of the Coulomb operator in Mulliken notation, and $c_{x}$ is a scalar that interpolates between pure semilocal density functionals $\left(c_{x}=0\right)$ and Hartree-Fock theory $\left(c_{x}=1, f^{\mathrm{xc}}=0\right)$. The vast majority of applications use the adiabatic approximation, ${ }^{58}$ which replaces the frequencydependent exchange-correlation kernel with its static limit. The excitation vector for state $n$ encodes the Kohn-Sham ground-to-excited state transition density matrix

$$
\gamma^{0 n}\left(x, x^{\prime}\right)=\sum_{i a}\left(X_{i a}^{n} \varphi_{i}(x) \varphi_{a}\left(x^{\prime}\right)+Y_{i a}^{n} \varphi_{a}(x) \varphi_{i}\left(x^{\prime}\right)\right)
$$

in terms of the Kohn-Sham orbitals $\varphi_{p} .{ }^{57}$ Eigenvectors of eqn (7) satisfy the orthonormalization condition

$$
\left\langle X^{n}, Y^{n}|\Delta| X^{m}, Y^{m}\right\rangle=\delta_{n m} .
$$

\subsection{Ground-to-excited-state derivative couplings}

First-order derivative couplings between the ground and an excited electronic state within the adiabatic approximation to TDDFT are obtained from a pole analysis of the frequencydependent linear response of the time-dependent Kohn-Sham wavefunction, ${ }^{33,59}$ and are computed together with excited-state gradients as

$$
\tau_{0 n}^{(\xi)}=\left\langle\mathbf{D}^{0 n, \mathrm{AO}^{(\xi)}}\right\rangle+\left\langle\mathbf{D}^{0 n, \mathrm{AO}} \mathbf{v}^{\mathrm{xc},(\xi)}\right\rangle-\left\langle\mathbf{W}^{0 n, \mathrm{AO}} \mathbf{S}^{(\xi)}\right\rangle+\left\langle\Gamma^{0 n, \mathrm{AO}} \mathbf{V}^{(\xi)}\right\rangle
$$

here, the superscript $(\xi)$ indicates partial differentiation, $\xi$ represents the nuclear coordinate of interest, the superscript $\mathbf{M}^{\mathrm{AO}} \equiv \mathbf{C M C}^{\dagger}$ indicates the quantity is expressed in the AO basis for molecular orbital coefficient matrix $\mathbf{C}, \mathbf{h}$ is the one-electron core Hamiltonian, $\mathbf{v}^{\mathrm{xc}}$ is the exchange-correlation potential, $\mathbf{S}$ is the overlap matrix, $\mathbf{D}^{0 n}$ is a generalized one-electron transition density, $\mathbf{W}^{0 n}$ is a generalized energy-weighted transition density, $\Gamma^{0 n}$ is the pair transition density, and $\mathbf{V}$ is the two-electron Coulomb operator defined with $V_{\mu \nu \lambda \kappa}=(\mu \nu \mid \lambda \kappa)$; greek indices denote AOs. At variance with ref. 59, we include in eqn (13) only translationally invariant terms, which is approximately equivalent to employing electron-translation factors. ${ }^{60,61}$

\subsection{State-to-state derivative couplings}

In analogy to the ground-to-excited state derivative couplings, state-to-state derivative couplings are defined through a pole analysis of the quadratic response function. The generator for the derivative coupling between excited states $n$ and $m$ is ${ }^{33}$

$$
G_{n m}\left(\mathbf{C}, \varepsilon \mid \gamma^{n m}, \mathbf{R}\right)=\left\langle X^{n}, Y^{n}|\Lambda(\mathbf{R})| X^{m}, Y^{m}\right\rangle / \Omega_{n m}+\left\langle\gamma^{n m, T} \mathbf{C}^{(0) \dagger} \mathbf{O}(\mathbf{R}) \mathbf{C}\right\rangle,
$$

where $O_{\mu \nu}(\mathbf{R}) \equiv\left\langle\chi_{\mu} \mid \chi_{\nu}(\mathbf{R})\right\rangle$ is a one-sided atomic orbital overlap integral, $\left|\chi_{\mu}\right\rangle$ is an atom-centered Gaussian basis function, the superscript (0) indicates that the quantity is fixed at the zerothorder solution, $\Omega_{n m} \equiv \Omega_{n}-\Omega_{m}, \gamma^{n m}$ is the 1-particle transition density matrix (1TDM) of the KS system, and dependence on the nuclear coordinates, $\mathbf{R}$, is now denoted explicitly.

Quadratic response theory dictates that the 1TDM in eqn (14) is obtained from

$$
\gamma^{n m, \mathrm{QR}}=\left(\begin{array}{cc}
-\left(\mathbf{X}^{n}\left(\mathbf{X}^{m}\right)^{T}+\mathbf{Y}^{n}\left(\mathbf{Y}^{m}\right)^{T}\right) & \mathbf{X}^{n m} \\
\left(\mathbf{Y}^{n m}\right)^{T} & \left(\mathbf{X}^{n}\right)^{T} \mathbf{X}^{m}+\left(\mathbf{Y}^{n}\right)^{T} \mathbf{Y}^{m}
\end{array}\right)
$$

where the off-diagonal blocks require the solution of a dynamic-polarizability-like equation,

$$
\left|X^{n m}, Y^{n m}\right\rangle=-\left(\Lambda-\Omega_{n m} \Delta\right)^{-1}\left|P^{n m}, Q^{n m}\right\rangle
$$

Explicit expressions for the right-hand-side (RHS) are provided in ref. 36 and supplied in the Appendix for completeness. However, within the adiabatic approximation to the exchangecorrelation kernel, the linear response operator in eqn (16) becomes singular when $\Omega_{n m}$ approaches any other excitation energy and thus the transition density diverges unphysically. ${ }^{33-36,62}$ Thus, we exclusively use derivative couplings from the pseudowavefunction approximation which is equivalent to ignoring the off-diagonal blocks of the 1TDM in eqn (14),

$$
\gamma^{n m, \mathrm{PW}}=\mathbf{T}^{n m}=\left(\begin{array}{cc}
\gamma_{\mathrm{oO}}^{n m, \mathrm{QR}} & \mathbf{0} \\
\mathbf{0} & \gamma_{\mathrm{vv}}^{n m, \mathrm{QR}}
\end{array}\right),
$$

where $\gamma_{\mathrm{oo}}^{n m, \mathrm{QR}}$ and $\gamma_{\mathrm{vv}}^{n m, \mathrm{QR}}$ are the occupied-occupied and virtualvirtual blocks of $\gamma^{n m, \mathrm{QR}}$, and $\mathbf{T}^{n m}$ is referred to as the unrelaxed 1TDM.

Regardless of the choice of 1TDM in eqn (14), an expression for the state-to-state derivative couplings including all Pulay terms is obtained by stationarizing the Lagrangian

$$
\begin{aligned}
L_{n m}\left[\mathbf{C}, \varepsilon, \mathbf{D}^{n m}, \overline{\mathbf{W}}^{n m}\right]= & G_{n m}\left(\mathbf{C}, \varepsilon \mid \gamma^{n m}, \mathbf{R}\right)+\left\langle\mathbf{D}^{n m, T}\left(\mathbf{C}^{\dagger} \mathbf{F C}-\varepsilon\right)\right\rangle \\
& -\left\langle\overline{\mathbf{W}}^{n m, T}\left(\mathbf{C}^{\dagger} \mathbf{S C}-\mathbf{I}\right)\right\rangle
\end{aligned}
$$

where $\varepsilon$ is a Lagrange multiplier assumed to be block-diagonal, $\mathbf{F}$ is the (density-matrix dependent) Fock matrix in atomic orbital (AO) representation, $\mathbf{D}^{n m}$ and $\overline{\mathbf{W}}^{n m}$ are Lagrange multipliers that require the molecular orbitals to satisfy the KS equation and enforce orthonormality, respectively, and $\langle\cdot\rangle$ indicates a trace. $\mathbf{D}^{n m}$ and $\overline{\mathbf{W}}^{n m}$ are determined by enforcing stationarity with respect to the remaining parameters,

$$
\left(\frac{\partial L_{n m}}{\partial \varepsilon}\right)=0,
$$

and

$$
\left(\frac{\partial L_{n m}}{\partial \mathbf{C}}\right)=\left(\frac{\partial L_{n m}}{\partial \mathbf{C}^{\dagger}}\right)=0 .
$$

Real orbitals are assumed only after taking derivatives such that fully general expressions are obtained. An outline of the derivation is provided in the Appendix. At the stationary point, the total derivative of $G_{n m}$ is obtained straightforwardly through a 
partial derivative of the Lagrangian,

$$
\begin{aligned}
& \frac{\mathrm{d} G_{n m}}{\mathrm{~d} \xi}= \frac{\partial L_{n m}}{\partial \xi} \equiv L_{n m}^{(\xi)} \\
&=\left\langle\mathbf{D}^{n m, \mathrm{AO}, T} \mathbf{h}^{(\xi)}\right\rangle+\left\langle\mathbf{D}^{n m, \mathrm{AO}, T} \mathbf{v}^{\mathrm{xc}(\xi)}\right\rangle \\
&-\left\langle\overline{\mathbf{W}}^{n m, \mathrm{AO}, T} \mathbf{S}^{(\xi)}\right\rangle+\left\langle\gamma^{n m, \mathrm{AO}, T} \mathbf{O}^{(\xi)}\right\rangle \\
&+\left\langle\Gamma^{n m, \mathrm{AO}, T} \mathbf{V}^{(\xi)}\right\rangle+\left\langle\Gamma^{n m, \mathrm{AO}, T} \mathbf{f}^{\mathrm{xc}(\xi)}\right\rangle \\
&=\left\langle\mathbf{D}^{n m, \mathrm{AO}, T} \mathbf{h}^{(\xi)}\right\rangle+\left\langle\mathbf{D}^{n m, \mathrm{AO}, T} \mathbf{v}^{\mathrm{xc}(\xi)}\right\rangle-\left\langle\mathbf{W}^{n m, \mathrm{AO}, T} \mathbf{S}^{(\xi)}\right\rangle \\
&+\left\langle\Gamma^{n m, \mathrm{AO}, T} \mathbf{V}^{(\xi)}\right\rangle+\left\langle\Gamma^{n m, \mathrm{AO}, T} \mathbf{f}^{\mathrm{xc}(\xi)}\right\rangle
\end{aligned}
$$

where $\Gamma^{n m}$ is the pair transition density and all other quantities are defined as in eqn (13). Eqn (22) follows from eqn (21) by introducing $S_{\mu \nu}^{(-\xi)}=\left\langle\chi_{\mu}^{(\xi)} \mid \chi_{\nu}\right\rangle-\left\langle\chi_{\mu} \mid \chi_{\nu}^{(\xi)}\right\rangle$, then recognizing that $\mathbf{O}^{(\xi)}=\frac{1}{2}\left(\mathbf{S}^{(\xi)}+\mathbf{S}^{(-\xi)}\right)$ such that the Hermitian part of $\gamma^{n m}$ can be combined with $\mathbf{W}^{n m}$,

$$
\mathbf{W}^{n m}=\overline{\mathbf{W}}^{n m}-\frac{1}{4}\left(\gamma^{n m}+\gamma^{n m, \dagger}\right) .
$$

The contractions involving $\mathbf{S}^{(-\xi)}$ with the anti-Hermitian part of $\gamma^{n m}$ can be neglected on physical grounds. ${ }^{60}$

\subsection{State-to-state derivative coupling implementation}

Eqn (22) was implemented into the program egrad, ${ }^{63}$ building on the existing implementation of excited-state gradients and ground-to-excited-state derivative couplings. The rate-determining steps are the computation of a user-specified set of excitations, eqn (7), the construction of the relaxed densities, eqn (16), and the final contraction of the effective densities with operator partial derivatives, eqn (22). First, a user-specified set of excited states are computed, then couplings are computed between all excitations in a user-specified subset of the original excitations. For each pair of excited states, one coupled-perturbed Kohn-Sham (CPKS) calculation is required (for derivative couplings within full quadratic response theory, this becomes a dynamic polarizability calculation). The right-hand-sides for all pairs of excited states in eqn (16) are computed simultaneously, together with the righthand-sides for excited-state gradients, ${ }^{63}$ using identical methodology as excited-state absorption calculations. ${ }^{36}$ Thus the state-tostate derivative coupling implementation achieves the same high resource-efficiency as shown for other quadratic response properties, ${ }^{36}$ up to the final contraction with operator derivatives. The required CPKS calculations were performed iteratively and simultaneously as described in ref. 36 and 64 with an integral driven ${ }^{65-69}$ nonorthonormal Krylov space block Davidson algorithm. ${ }^{70}$ Finally, for each operator type (e.g., h), contractions between its partial derivatives $\left(\mathbf{h}^{(\xi)}\right)$ and all effective densities-including effective densities needed to compute ground- and excited-state gradients-were performed together such that the partial derivatives only need to be computed once.

Our implementation permits the computation of derivative couplings between excited-states computed with spin-restricted
Kohn-Sham (RKS) or spin-unrestricted KS (UKS) orbitals, with or without the resolution-of-the-identity approximation for the Coulomb integrals, ${ }^{71}$ with and without applying the TammDancoff approximation, ${ }^{72}$ and for non-hybrid and hybrid semilocal density functionals, or time-dependent Hartree-Fock. Transition densities and RHSs between excited states were verified by reconstructing the dynamic hyperpolarizability from the exact TDDFT sum-over-states expressions. The final derivative couplings were verified against finite difference results. ${ }^{33}$

\section{Excited-state deactivation of thymine}

\subsection{Prior results}

Experimentally, three decay channels of UV-photoexcited thymine have been distinguished on the basis of gas phase femtosecond pump-probe transient ionization spectroscopy, ${ }^{37,38,40-43}$ femtosecond time-resolved photoelectron spectroscopy (TRPES), ${ }^{39}$ and ultrafast X-ray Auger, ${ }^{44}$ see Table 1: (i) a prompt signal with a time constant near 100-200 fs (ii) a fast signal with time constant of 5.1-7 ps and (iii) a slow decay conventionally attributed to intersystem crossing that is longer than 100 ps. Although mechanistic details could be inferred for aqueous thymine photodynamics using UV resonance Raman spectroscopy, ${ }^{73,74}$ the decay mechanism of UV-photoexcited thymine in the gas phase is experimentally difficult to probe.

The photodynamics of nucleic acids and nucleobases have been studied extensively computationally ${ }^{53}$ using a range of different approaches, including characterization of the minimum energy pathways through conical intersections ${ }^{42,46,47,75,76}$ and direct dynamics simulations. ${ }^{43,48-52}$ Here, we focus on on-the-fly dynamical simulations of photoexcited thymine and refer the interested reader to a several extensive reviews for additional details. ${ }^{4,53}$ Previously reported on-the-fly simulations of thymine photodynamics can be broadly categorized according to the electronic structure methods used to power the dynamics, see Table 2 . To date, the most advanced on-the-fly NAMD simulations have used semiempirical models, ${ }^{77} \mathrm{MCSCF},{ }^{43,49-51}$ and $\mathrm{ADC}(2)$ theory, with partially conflicting conclusions.

In particular, there appears to be no general consensus on the deactivation mechanism for photoexcited thymine with an excitation wavelength of $266 \mathrm{~nm}$, near absorption onset.

Table 1 Summary of experimental time constants for ultrafast deactivation of UV-photoexcited thymine ${ }^{37-42}$ obtained with experimental techniques pump-probe transient ionization (PPTI), pump-probe resonant ionization (PPRI), pump-probe ionization spectroscopy (PPIS), and time-resolved photoelectron spectroscopy (TRPES)

\begin{tabular}{lllll}
\hline & Component & & & \\
\cline { 2 - 5 } Measurement & Ultrashort (fs) & Prompt (fs) & Fast (ps) & Slow (ns) \\
\hline PPTI $^{37}$ & & & 6.4 & $>100$ \\
TRPES $^{39}$ & $<50$ & 490 & 6.4 & \\
PPRI $^{38}$ & & 105 & 5.12 & \\
PPIS $^{40,41}$ & & 130 & 6.5 & $>1$ \\
PPIS $^{42}$ & 100 & 7 & \\
X-ray Auger & & 200 & &
\end{tabular}


Table 2 Summary of computed time constants for ultrafast deactivation of UV-photoexcited thymine with available confidence intervals shown in parentheses in comparison to experimental results (see Table 1 for details)

\begin{tabular}{|c|c|c|c|c|}
\hline & \multicolumn{4}{|l|}{ Component } \\
\hline Semiempirical $^{48}$ & $420 \mathrm{fs}$ & $\mathrm{S}_{1} \rightarrow \mathrm{S}_{0}$ & & $\mathrm{~S}_{0} \rightarrow \mathrm{S}_{0, \mathrm{eq}}$ \\
\hline $\operatorname{ADC}(2)^{52}$ & $250 \mathrm{fs}$ & $\mathrm{S}_{2} \rightarrow \mathrm{S}_{1}$ & $420 \mathrm{fs}$ & $\mathrm{S}_{1} \rightarrow \mathrm{S}_{0}$ \\
\hline PBE0/SVP (this work) & $153 \mathrm{fs}(147-162 \mathrm{fs})$ & $\mathrm{S}_{2} \rightarrow \mathrm{S}_{1}$ & 13.9 ps (10.4-21 ps) & $\mathrm{S}_{1} \rightarrow \mathrm{S}_{0}$ \\
\hline PBE0/SVPD (this work) & $110 \mathrm{fs}(82-128 \mathrm{fs})$ & $\mathrm{S}_{2} \rightarrow \mathrm{S}_{1}$ & 20.5 ps (11.6-49 ps) & $\mathrm{S}_{1} \rightarrow \mathrm{S}_{0}$ \\
\hline
\end{tabular}

Semiempirical models such as multireference configuration interaction with the OM2 Hamiltonian ${ }^{77}$ (OM2/MRCI) - which are parametrized to reproduce energies near ground state geometries and can thus become unreliable far from the Franck-Condon region such as near conical intersections-predict an overall excited-state lifetime of $437 \mathrm{fs}$, about one order of magnitude faster than that observed experimentally. ${ }^{48}$ Furthermore, the predicted $\mathrm{S}_{2}$ lifetime of $17 \mathrm{fs}$ and $\mathrm{S}_{1}$ lifetime of $420 \mathrm{fs}$ lead to the interpretation that the ultrashort signal corresponds to internal conversion $\mathrm{S}_{2} \rightarrow \mathrm{S}_{1}$, the prompt signal corresponds to internal conversion to the ground state $\left(S_{1} \rightarrow S_{0}\right)$ and the fast component corresponds to thermalization on the ground state. ${ }^{48}$

MCSCF, especially the state-averaged complete active space self-consistent field (SA-CASSCF) variant, is commonly used for NAMD simulations because, as a multireference method, it correctly recovers the topology of potential energy surface crossings. ${ }^{78}$ However, CASSCF lacks dynamical correlation and can therefore overestimate the absorption onset and the $\mathrm{S}_{2}-\mathrm{S}_{1}$ energy gap by up to $1.5 \mathrm{eV}$ in thymine relative to more accurate methods. ${ }^{51}$ Thymine's photochemistry has been simulated using CASSCF by several different groups ${ }^{43,49-51}$ with a qualitatively consistent picture emerging in which photoexcited thymine relaxes from the Franck-Condon (FC) geometry to a minimum on the $S_{2}$ potential energy surface with a time constant of 100-200 fs thus giving rise to the prompt signal. The $S_{2}$ state then decays to the $S_{1}$ state with an estimated time constant of 2.6-5 ps and $S_{1}$ also exhibits a lifetime of several ps although precise estimates have not been provided. From this, the fast signal is assigned to the compound $\mathrm{S}_{2, \min } \rightarrow \mathrm{S}_{1} \rightarrow \mathrm{S}_{0}$ deactivation. The relatively long $S_{2}$ lifetime has been attributed to a small but significant energetic barrier of $\approx 0.25 \mathrm{eV}$ $\left(5.7 \mathrm{kcal} \mathrm{mol}^{-1}\right.$ ) between the $\mathrm{S}_{2}$ minimum and the $\mathrm{S}_{2} / \mathrm{S}_{1}$ conical intersection. ${ }^{75}$ However, this trapping on the $S_{2}$ state surface was called into question based on ultrafast X-ray Auger experiments that observe a distinct electronic relaxation within $200 \mathrm{fs}^{44}$ Furthermore, the barrier all but disappears with the inclusion of dynamic correlation. For example, the barrier falls to $0.05 \mathrm{meV}$ $\left(1 \mathrm{kcal} \mathrm{mol}^{-1}\right)$ using multistate complete active-space secondorder perturbation theory (MS-CASPT2). ${ }^{75}$

To test the effect of dynamic correlation on the ensuing dynamics, Stojanovic et al. ${ }^{52}$ used the ADC(2) method. ADC(2) is complementary to CASSCF in that dynamic correlation is explicitly included to second-order, but being a single-reference method, $\operatorname{ADC}(2)$ is sensitive to strong static correlation due to near-degeneracy of the ground state. In particular, although stateto-state conical intersections are correctly reproduced, groundto-excited-state intersections have the wrong dimensionality. ${ }^{79}$ However, this may not be a cause for concern; preliminary investigations have shown that mixed quantum-classical trajectories are relatively insensitive to the dimensionality of the surface crossing, producing qualitatively similar results for different excited-state surface topologies. ${ }^{80}$ As opposed to the several picosecond $\mathrm{S}_{2}$ lifetime found with CASSCF dynamics, $\operatorname{ADC}(2)$ based dynamics indicate that following relaxation to the $S_{2}$ minimum in $100 \mathrm{fs}$, the majority of trajectories (84\%) decay to $\mathrm{S}_{1}$ within $250 \mathrm{fs}$. Out of these trajectories, $83 \%$ ( $70 \%$ of total trajectories) decay to the ground state within $400 \mathrm{fs}$. This lead to the conclusion that the prompt signal corresponds to decay to $S_{2}$ with the fast signal corresponding to $S_{1} \rightarrow S_{0}$ deactivation, but the computed rate for the latter reaction is approximately one order of magnitude too fast.

\subsection{Computational details}

Initial conditions were drawn randomly from a $9.5 \mathrm{ps}$ ground state ab initio molecular dynamics (AIMD) simulation at $500 \mathrm{~K}$ after a 0.5 ps equilibration period. The molecular dynamics were propagated with the leapfrog Verlet algorithm with an 80 a.u. $(\approx 1.935 \mathrm{fs})$ time step. The TPSS density functional ${ }^{81}$ with D3 dispersion corrections ${ }^{82}$ and Becke-Johnson (BJ) damping parameters ${ }^{83}$ and the def2-SVP basis set $^{84}$ was used to compute the ground state energy at each time step. For these AIMD simulations, the resolution-of-the-identity (RI) was used to approximate the two-electron Coulomb integrals, ${ }^{71} \mathrm{~m} 3$ grids with quadrature weight derivatives were used for functional integrations, and self-consistent field (SCF) convergence thresholds were set to $10^{-7} E_{\mathrm{h}}$ (scfconv 7).

NAMD simulations were initiated on the bright $S_{2}$ surface with initial electronic density matrix $\sigma_{n m}=\delta_{n, 2} \delta_{m, 2}$ and used the PBE0 density functional ${ }^{85}$ with D3-BJ dispersion corrections ${ }^{82,83}$ and either split valence (def2-SVP) ${ }^{84}$ or property-optimized (def2SVPD $)^{86}$ basis sets. Excited states were computed within the Tamm-Dancoff approximation (TDA). ${ }^{72}$ Molecular dynamics were propagated with the leapfrog Verlet algorithm and a time step of 40 a.u. $(0.9676 \mathrm{fs})$. For the NAMD simulations, size 4 grids with quadrature weight derivatives were used for functional integrations, SCF convergence thresholds were set to $10^{-9} E_{\mathrm{h}}$ (scfconv 9), and excited states were converged to a residual norm of less than $10^{-7}$ (rpaconv 7). As for $\operatorname{ADC}(2)$, conical 
intersections between the ground state and an excited state have incorrect dimensionality with TDDFT. ${ }^{78}$ To avoid potential associated instabilities, trajectories are forced to hop to the ground state if the first excitation energy, $\Omega_{1}$, falls below $0.5 \mathrm{eV}$. In total, we simulated 200 independent trajectories for a combined $650 \mathrm{ps}$ of simulation time.

All uncertainties indicated here correspond to $95 \%$ confidence intervals (CI) estimated from bootstrap sampling with 10000 samples. ${ }^{87}$ Bootstrap sampling is a powerful resampling technique to estimate uncertainties in properties computed from a set of measurements of a stochastic process, i.e., without assuming an analytical distribution. Bootstrap sampling is therefore well-suited to estimating errors from NAMD trajectories where no information about the underlying statistical process is available. ${ }^{88}$ To obtain the bootstrap confidence interval of, for example, the mean value of a given set of $N_{\text {measure }}$ measurements of a stochastic process, $N_{\text {bootstrap }}$ replica samples are generated, the mean is computed for each replica sample, and confidence intervals are chosen to compactly contain the specified proportion of all replica means. Replica samples are generated by randomly selecting $N_{\text {measure }}$ measurements with replacement (meaning duplicates are likely) out of the original $N_{\text {measure }}$ measurements.

\subsection{Thymine excited states}

The lowest-lying excited state $\left(S_{1}\right)$ of thymine is a dark $n-\pi^{*}$ transition dominated by a single occupied-to-virtual transition, see Fig. 1, in which the dominant natural transition orbitals (NTOs) (singular value 0.997 with PBE0/def2-SVP) correspond to a HOMO-1 (highest occupied molecular orbital) to LUMO (lowest unoccupied molecular orbital) transition. The second excited-state $\left(\mathrm{S}_{2}\right)$, on the other hand, is a bright $\pi-\pi^{*}$ transition mostly characterized by a single HOMO-to-LUMO orbital transition (singular value 0.91). Using PBE0, we found little to no dependence of the $\mathrm{S}_{1}$ vertical excitation energy on the basis set-4.85 eV with def2-SVP compared to $4.83 \mathrm{eV}$ with def2SVPD-and a small but notable dependence of the $S_{2}$ vertical
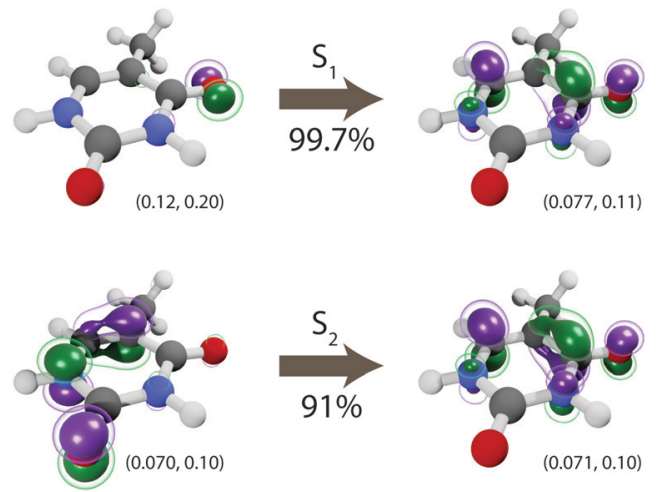

Fig. 1 Dominant natural transition orbitals (NTOs) for the $S_{1}\left(n-\pi^{\star}\right)$ and $\mathrm{S}_{2}\left(\pi-\pi^{\star}\right)$ electronic states. The corresponding singular value is shown under the arrow. For each NTO, isovalues are shown below the corresponding orbital and chosen to contain approximately 25\% (inner) and 50\% (outer) of the total orbital density. All excitations at the Franck-Condon geometry.
Table 3 Computed vertical excitation energies for the first two singlet excited states of thymine compared to experimental results from gasphase electron energy loss (EEL) spectroscopy (band maxima)

\begin{tabular}{lll}
\hline Method & $\mathrm{S}_{1}(\mathrm{eV})$ & $\mathrm{S}_{2}(\mathrm{eV})$ \\
\hline PBE0/SVP (this work) & 4.85 & 5.44 \\
PBE0/SVPD (this work) & 4.83 & 5.24 \\
ADC $(2)^{52}$ & 4.56 & 5.06 \\
MS-CASPT2 $^{50}$ & 5.09 & 5.09 \\
MS-CASPT2 $^{43}$ & 5.23 & 5.44 \\
CASSCF $^{50}$ & 5.31 & 7.12 \\
EEL spectroscopy & & $4.95,{ }^{89} 4.9,,^{90} 4.96^{91}$
\end{tabular}

excitation energy on the basis set-5.44 eV with def2-SVP compared to $5.24 \mathrm{eV}$ with def2-SVPD. No further significant change was observed when moving to even larger basis sets: with def2-TZVPPD, the vertical excitation energies are $4.82 \mathrm{eV}$ and $5.21 \mathrm{eV}$, respectively. For all of the basis sets considered here, the excitation energies computed using PBE0 at the ground state geometry agree well with excitation energies measured experimentally and computed by other methods, see Table 3.

\subsection{Dynamics}

All simulated trajectories exhibit similar qualitative behavior exemplified by the sample trajectory shown in Fig. 2: after being initiated on the $\mathrm{S}_{2}$ state, thymine rapidly undergoes internal conversion to the $\mathrm{S}_{1}$ state (in all cases occurring in less than $250 \mathrm{fs}$ ) followed by a slower decay to $S_{0}$ on the order of several ps.

Since the detailed nonadiabatic dynamics are expected to be highly sensitive to the energy difference between different excited states-which is itself sensitive to the basis set-we first assessed the difference between observed dynamics using both def2-SVP and def2-SVPD basis sets. 100 trajectories were simulated for each basis set using FSSH and trajectories were propagated for 5 ps using def2-SVP and 1.5 ps using def2-SVPD. Fig. 3 compares the evolution of the excited state populations along these trajectories. Excited state populations in Fig. 3 were computed as $p_{k}(t)=N_{k}(t) / N_{\text {traj }}$ where $N_{k}(t)$ is the number of trajectories with state $k$ as active state at time $t$.

The $S_{2}$ decay with def2-SVPD was found to be significantly faster than with def2-SVP: with def2-SVP, the half-life of the $\mathrm{S}_{2}$

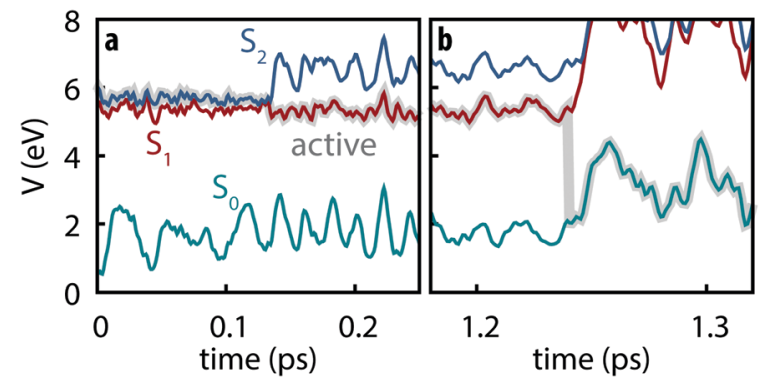

Fig. 2 Example trajectory showing (a) the subpicosecond internal conversion from $S_{2}$ to $S_{1}$ followed by (b) slow decay to $S_{0}$. 

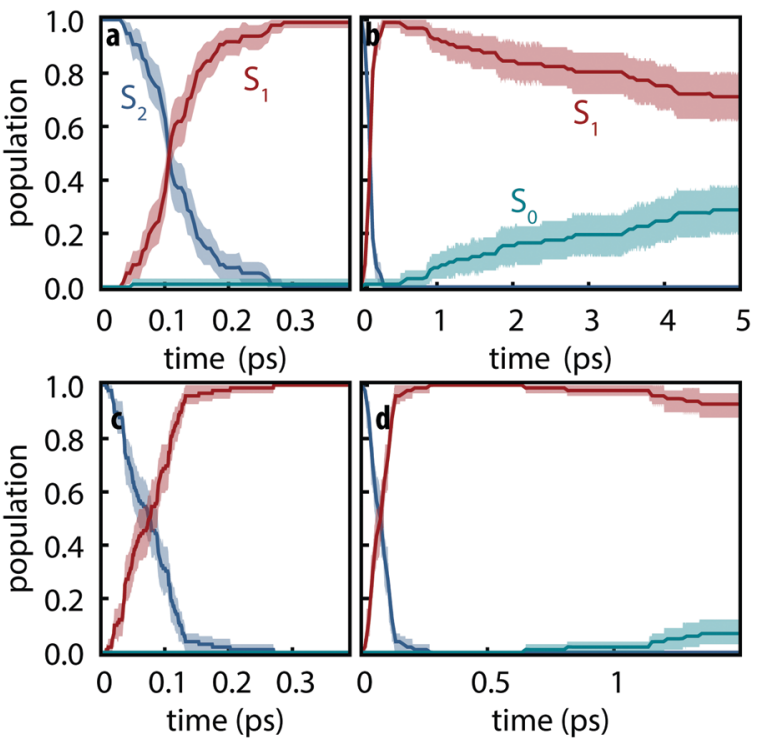

Fig. 3 Excited-state populations measured over trajectory swarms using ( $a$ and $b$ ) def2-SVP and ( $c$ and d) def2-SVPD basis sets. Shaded regions show the 95\% bootstrap confidence interval. Panels (a) and (c) show the short time behavior while panels (b) and (d) show the picosecond behavior. Note the different time scales in (b) and (d).

state is $106 \mathrm{fs}$ (CI: 102-112 fs) whereas the half-life with def2SVPD was 76 fs (CI: 57-89 fs). The observed increased rate can be attributed to the reduced energy gap at the Franck-Condon geometry between $\mathrm{S}_{1}$ and $\mathrm{S}_{2}, \Delta E_{21}=E_{\mathrm{S}_{2}}-E_{\mathrm{S}_{1}}$, which is $0.59 \mathrm{eV}$ at def2-SVP and $0.41 \mathrm{eV}$ at def2-SVPD, i.e., the energy gap and the half-life both reduce by $\approx 70 \%$. On the other hand, since $\Delta E_{10}$ is only slighty affected by the basis set, no significant change in the $\mathrm{S}_{1}$ lifetime is expected or observed. After $1.5 \mathrm{ps,}$ the proportion of trajectories on the ground state have strongly overlapping confidence interval ranges (6-18\% with def2-SVP vs. $3-12 \%$ with def2-SVPD). We therefore conclude that after the ultrafast decay to $S_{1}$, def2-SVP and def2-SVPD recover essentially identical dynamics and we thus continue our analysis with only the def2-SVP results.

The initial dynamics were quantified in terms of four structural parameters, the $\mathrm{C}-\mathrm{O}$ bond distance of the carbonyl on which the $\mathrm{S}_{1}$ hole is located, the C-Me (where Me is methyl), the $\mathrm{C}-\mathrm{C}$ bond distance for the carbon atoms in the ring connecting the active carbonyl and the $\mathrm{CMe}$, and the ring puckering amplitude, $Q$, computed using the Cremer-Pople coordinates. ${ }^{92}$ Fig. 4 summarizes the dynamics simulated using FSSH and depicts the coordinates involved. Immediately upon photoexcitation, the $\mathrm{C}-\mathrm{O}$ bond lengthens and the $\mathrm{C}-\mathrm{C}$ bond shortens, both of which are consistent with observed shifts in the oxygen Auger spectrum ${ }^{44}$ and with the interpretation that the particle (virtual) NTO of the $\mathrm{S}_{2}$ excitation exhibits a bonding-like $\pi$ overlap on the $\mathrm{C}-\mathrm{C}$ bond in the ring and an antibonding-like structure on the carbonyl. In addition, the puckering amplitude more than doubles from the equilibrium value of about $0.08 \AA$ to $0.23 \AA$ within 120 fs. However, we find no significant difference in the degree of planarity between the ground state and $\mathrm{S}_{1}$, and thus attribute the increased degree of

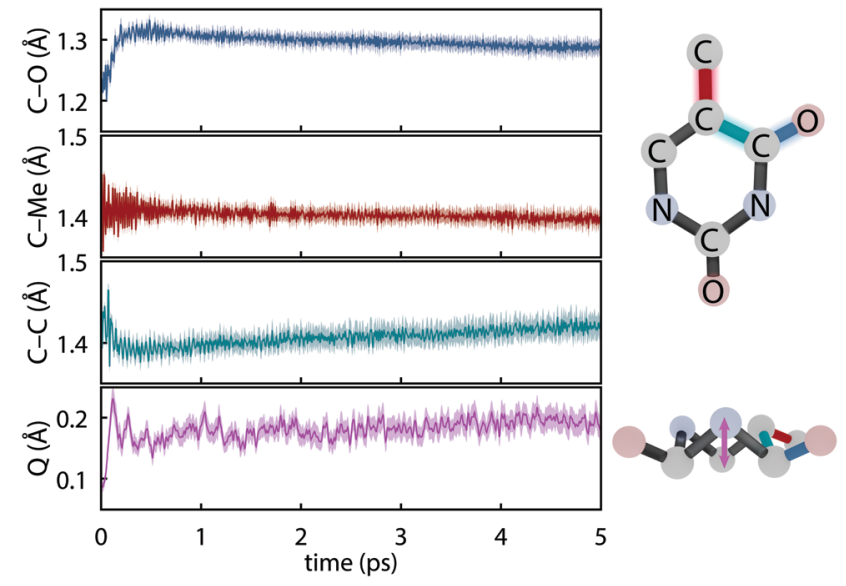

Fig. 4 Major geometrical changes measured over trajectory swarms accompanying ultrafast dynamics simulated with def2-SVP and FSSH. Shaded regions show the $95 \%$ bootstrap confidence interval. (a) $\mathrm{C}-\mathrm{O}$ bond distance (b) C-Me bond distance (c) aromatic C-C bond distance and $(d)$ the puckering amplitude, $Q .{ }^{92}$

puckering to the increased vibrational energy resulting from the nonradiative decay.

The simulated rates for the two electronic decays $\left(\mathrm{S}_{2} \rightarrow \mathrm{S}_{1}\right.$ and $S_{1} \rightarrow S_{0}$ ) are characterized as single exponential decays from which two lifetimes are defined according to

$$
p_{2}(t>0) \approx \mathrm{e}^{-t / \tau_{2}},
$$

and

$$
p_{0}(t>0) \approx 1-\mathrm{e}^{-t / \tau_{0}}
$$

where $\tau_{2}$ corresponds to the $S_{2} \rightarrow S_{1}$ decay channel and $\tau_{0}$ corresponds to the rise of the ground state or conversely the total excited state lifetime. $\tau_{2}$ is estimated through the $S_{2}$ halflife, $t_{\mathrm{S}_{2}, 1 / 2}$, according to

$$
\tau_{2}=t_{\mathrm{S}_{2}, 1 / 2} / \ln 2,
$$

while $\tau_{0}$ is similarly estimated from the final value in the simulation using

$$
\tau_{0}=-t_{\mathrm{f}} / \ln \left(1-p_{0, \mathrm{f}}\right),
$$

where $t_{\mathrm{f}}$ and $p_{0, \mathrm{f}}=p_{0}\left(t_{\mathrm{f}}\right)$ are the time and population used to estimate the rate.

Using the set of def2-SVP simulations, we find the $\mathrm{S}_{2}$ lifetime to be $\tau_{2}=153 \mathrm{fs}$ (CI: 147-162 fs) which agrees well with the 100-200 fs lifetimes measured for the prompt signal. Consequently, we assign the prompt signal to the $S_{2} \rightarrow S_{1}$ decay. The total excited state lifetime was found to be $13.9 \mathrm{ps}$ (CI: 10.4-21 ps), which agrees well (within a factor of 2) with the 5-7 ps lifetimes measured for the fast component such that we assign the fast signal to correspond to the $\mathrm{S}_{1} \rightarrow \mathrm{S}_{0}$ decay.

\subsection{Electronic populations and surface hopping}

Electronic populations within FSSH and many of its variants are ambiguously defined ${ }^{93}$ because the FSSH algorithm refers to two distinct effective electronic wavefunctions: (i) the auxiliary 


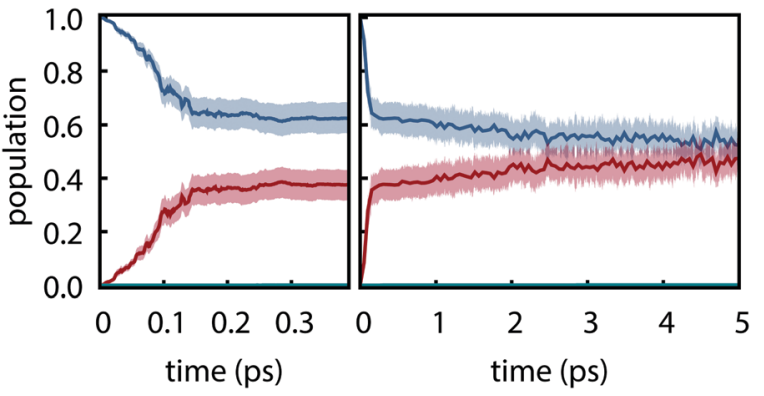

Fig. 5 Electronic populations computed from the auxiliary electronic density matrix for trajectories computed using PBE0-D3/def2-SVP and FSSH. Shaded regions show the $95 \%$ bootstrap confidence interval. (a) Short time and (b) long time behavior.

wavefunction, which is propagated using eqn (2) and (ii) the active wavefunction which is determined from the evolution of the auxiliary wavefunction and from which the potential energy and nuclear forces are computed. The preceding analysis follows the original prescription ${ }^{8}$ in which only the active wavefunction (i.e. the active surface) is used to determine electronic populations although other prescriptions have been proposed. ${ }^{94}$ However, it is important to examine the auxiliary electronic populations because they indirectly dictate the behavior of the active wavefunction and because they are central to the original motivation for hopping probabilities.

Electronic populations using the auxiliary wavefunction indicate negligible nonradiative decay from $\mathrm{S}_{1}$; instead, the populations slowly evolve towards a near equal mixture of $S_{1}$ and $S_{2}$ (see Fig. 5). This leads to a starkly different interpretation of the reactivity-that $S_{1}$ and $S_{2}$ are equally populated on the picosecond timescale-that is not supported by any experimental evidence. This is concerning given that the FSSH hopping probabilty was derived to satisfy the constraint that the swarm-based populations, i.e., populations computed through

$$
p_{k}(t)=\left\langle N_{k}\right\rangle(t) / N_{\text {traj }}
$$

where $\left\langle N_{k}\right\rangle(t)$ is the number of trajectories with active surface $k$ at time $t$ and $N_{\text {traj }}$ is the total number of trajectories, statistically match the auxiliary populations. ${ }^{8}$ It thus appears that physically valid results for thymine are obtained even though a central assumption underlying surface hopping is violated. These results imply that efforts to adjust the surface hopping criterion to better enforce the correspondence between swarm-based populations and the auxiliary electronic density matrix could adversely affect accuracy for the present system. ${ }^{95}$

Our observation is consistent with previous work on multistate $\mathrm{FSSH}^{96}$ and the fact that the total energy of a trajectory and all properties, including electronic populations, are best defined in terms of an energy derivative: ${ }^{97}$

$$
\sigma_{n m}^{\text {effective }}(t)=\frac{\partial\langle E\rangle(t)}{\partial H_{m n}(t)},
$$

where $E(t)$ is the trajectory energy which is defined as the nuclear kinetic energy plus the electronic energy of the active state.

\section{Conclusions}

Building on resource-efficient methodology previously developed for ground states, excited states, and quadratic response properties, the implementation of state-to-state derivative couplings reported here enables multistate nonadiabatic molecular dynamics simulations using hybrid-TDDFT for molecules with up to $\sim 100$ atoms and $\sim 10$ ps simulation times on single workstation nodes. For the first three singlet states of thymine, each step of an on-the-fly NAMD simulation including all couplings requires only a factor of 4.3 more CPU time than the corresponding ground-state AIMD simulation. As opposed to finite-difference approaches, the entire nonadiabatic coupling vector is obtained, enabling correct rescaling of velocities. The single Slater-determinant model for excited states corresponds to a zeroth-order noniterative approximation using unit excitation vectors and neglecting the Hartree, exchange, and correlation contributions to the excitation energy. Whether the resulting modest gain in efficiency is worthwhile in view of the loss of accuracy caused by neglect of configuration interaction appears questionable. Our implementation will be publicly released through Turbomole. ${ }^{98,99}$ All NAMD trajectory data are available through a Creative Commons license. ${ }^{100}$

Straightforward application of the present hybrid TDDFT-SH methodology to UV-photoexcited thymine semiquantitatively recovers experimental excited-state lifetimes. In particular, the TDDFT-SH lifetimes of 147-162 fs for $\mathrm{S}_{2}$ and $10.4-20.1 \mathrm{ps}$ for $\mathrm{S}_{1}$ agree well with the experimentally observed ones of 100-200 fs and 5-7 ps. The rapid $S_{2}$ decay is barrierless and proceeds through a conical intersection between $\mathrm{S}_{2}$ and $\mathrm{S}_{1}$ close to the Franck-Condon region, whereas the $\mathrm{S}_{1}$ decay is much slower. Thus, our results strongly support the $\mathrm{S}_{1}$ trapping hypothesis. ${ }^{43}$

For thymine deactivation, TDDFT using standard hybrid functionals yields significantly better agreement with experiment than MCSCF and ADC(2). While this result could be coincidential, it is consistent with the good performance of hybrid TDDFT for vertical excitation energies of thymine, as well as a mounting body of evidence suggesting that hybrid TDDFT has favorable cost-to-performance characteristics for a wide range of systems. ${ }^{13,16,20,25,26,101-108}$ Due to its resource efficiency, TDDFT-SH is particularly suitable for large-scale and exploratory applications. The striking discrepancy between electronic and trajectory-averaged populations for the slow decay of the $\mathrm{S}_{1}$ state of thymine may warrant further investigation. The current approach circumvents but does not fundamentally eliminate the divergences of inexact response theory in state-tostate properties. ${ }^{34,62}$

\section{Conflicts of interest}

Principal investigator Filipp Furche has an equity interest in Turbomole $\mathrm{GmbH}$. The terms of this arrangement have been reviewed and approved by the University of California, Irvine, in accordance with its conflict of interest policies. 


\section{Appendix: derivation of state-to-state derivative coupling}

We parametrize the state-to-state 1TDM in eqn (14) as

$$
\gamma^{n m}=\mathbf{T}^{n m}+c_{\mathrm{QR}}\left(\begin{array}{cc}
\mathbf{0} & \mathbf{X}^{n m} \\
\left(\mathbf{Y}^{n m}\right)^{T} & \mathbf{0}
\end{array}\right),
$$

where $\mathbf{T}^{n m}$ is defined in eqn (17), and $c_{\mathrm{QR}}$ is a constant that allows writing equations simultaneously for derivative couplings from quadratic response $\left(c_{\mathrm{QR}}=1\right)$ and pseudowavefunction $\left(c_{\mathrm{QR}}=0\right)$ approaches. For convenience, we define the linear transformations

$$
\begin{aligned}
H_{p q}^{+}[\mathbf{M}]= & \sum_{r s}\left[2(p q \mid r s)+2 f_{p q, r s}^{\mathrm{xc}}\right. \\
& \left.-c_{x}[(p s \mid q r)+(p r \mid q s)]\right] M_{r s}, \\
H_{p q}^{-}[\mathbf{M}]= & c_{x} \sum_{r s}[(p s \mid q r)-(p r \mid q s)] M_{r s},
\end{aligned}
$$

as well as the double contraction with the density functional hyperkernel,

$$
\begin{gathered}
g_{p q}^{\mathrm{xc}}\left[\mathbf{M}, \mathbf{M}^{\prime}\right]=\sum_{r s t u} g_{p q, r s, t u}^{\mathrm{xc}} M_{r s} M_{t u}^{\prime}, \\
\left.g^{\mathrm{xc}}\left(x, x^{\prime}, x^{\prime \prime}\right) \approx \frac{\delta^{3} E_{\mathrm{xc}}[\rho]}{\delta \rho(x) \delta \rho\left(x^{\prime}\right) \delta \rho\left(x^{\prime \prime}\right)}\right|_{\rho=\rho_{0}} .
\end{gathered}
$$

In addition, we use the following shorthand definitions:

$$
\begin{gathered}
\mathbf{R}^{ \pm n} \equiv \mathbf{X}^{n} \pm \mathbf{Y}^{n}, \\
\mathbf{M}^{ \pm} \equiv \frac{1}{2}\left(\mathbf{M} \pm \mathbf{M}^{T}\right), \\
H_{p q}^{ \pm n} \equiv H_{p q}^{ \pm}\left[\mathbf{R}^{ \pm n}\right], \\
\bar{\Omega}_{n m} \equiv \frac{1}{2}\left(\Omega_{n}+\Omega_{m}\right) .
\end{gathered}
$$

The orbital relaxation (off-diagonal) contributions to $\gamma^{\mathrm{nm}}$ require solution of

$$
\left|X^{n m}, Y^{n m}\right\rangle=-\left(\Lambda-\Omega_{n m} \Delta\right)^{-1}\left|P^{n m}, Q^{n m}\right\rangle
$$

with the right-hand-sides

$$
\begin{aligned}
(P+Q)_{i a}^{n m}= & -\frac{1}{2} \sum_{j}\left[R_{j a}^{+m} H_{j i}^{+n}+R_{j a}^{-m} H_{j i}^{-n}+(n \leftrightarrow m)\right] \\
& +\frac{1}{2} \sum_{b}\left[R_{i b}^{+m} H_{a b}^{+n}+R_{i b}^{-m} H_{a b}^{-n}+(n \leftrightarrow m)\right] \\
& +H_{i a}^{+}\left[\mathbf{T}^{+n m}\right]+2 g_{i a}^{\mathrm{xc}}\left[R^{+n}, R^{+m}\right],
\end{aligned}
$$

$$
\begin{aligned}
(P-Q)_{i a}^{n m}= & +\frac{1}{2} \sum_{j}\left[R_{j a}^{-m} H_{j i}^{+n}+R_{j a}^{+m} H_{j i}^{-n}-(n \leftrightarrow m)\right] \\
& -\frac{1}{2} \sum_{b}\left[R_{i b}^{-m} H_{a b}^{+n}+R_{i b}^{+m} H_{a b}^{-n}-(n \leftrightarrow m)\right] \\
& -H_{i a}^{-}\left[\mathbf{T}^{-n m}\right],
\end{aligned}
$$

where $(n \leftrightarrow m)$ signifies repeating the terms within the same bracket with $n$ and $m$ interchanged.

The Lagrange multiplier $\mathbf{D}^{n m}$ can be parametrized as

$$
\mathbf{D}^{n m}=\Omega_{n m}{ }^{-1}\left(\mathbf{T}^{n m}+\mathbf{Z}^{n m}\right),
$$

where the diagonal blocks $\left(\Omega_{n m}{ }^{-1} \mathbf{T}^{n m}\right)$ follow from eqn (19) and $\mathbf{Z}^{n m}$ is off-diagonal only. Next, expressions for $\mathbf{Z}^{n m}$ are obtained by enforcing stationarity with respect to orbitals. We define $L^{(p q)} \equiv \sum_{\mu} \frac{\partial L}{\partial C_{\mu p}} C_{\mu q}$ and $L^{\overline{(p q)}} \equiv \sum_{\mu} \frac{\partial L}{\partial C_{\mu q}{ }^{*}} C_{\mu q}{ }^{*}$ such that

$$
\begin{aligned}
\left\langle\mathbf{D}^{n m, T} \mathbf{C}^{\dagger} \mathbf{F C}\right\rangle^{(a p)} & =D_{p a}^{n m} \varepsilon_{p} \\
\left\langle\mathbf{D}^{n m, T} \mathbf{C}^{\dagger} \mathbf{F C}\right\rangle^{\overline{(a p)}} & =D_{a p}^{n m} \varepsilon_{p} .
\end{aligned}
$$$$
\left\langle\overline{\mathbf{W}}^{n m, T} \mathbf{C}^{\dagger} \mathbf{S C}\right\rangle^{(p q)}=\left\langle\overline{\mathbf{W}}^{n m, T} \mathbf{C}^{\dagger} \mathbf{S C}\right\rangle^{\overline{(q p)}}=\bar{W}_{q p}^{n m}
$$$$
\left\langle\mathbf{D}^{n m, T} \mathbf{C}^{\dagger} \mathbf{F C}\right\rangle^{(i p)}=D_{p i}^{n m} \varepsilon_{p}+\sum_{r s}[(r s \mid p i)-(r p \mid i s)] D_{r s}^{n m}
$$$$
\left\langle\mathbf{D}^{n m, T} \mathbf{C}^{\dagger} \mathbf{F C}\right\rangle^{\overline{(i p)}}=D_{i p}^{n m} \varepsilon_{p}+\sum_{r s}[(r s \mid i p)-(r i \mid p s)] D_{r s}^{n m}
$$

With this, the orbital stationarity conditions become

$$
\begin{aligned}
& L^{(i p)}=G_{n m}^{(i p)}+D_{p i}^{n m} \varepsilon_{p}+\sum_{r s}[(r s \mid p i)-(r p \mid i s)] D_{r s}^{n m}-\bar{W}_{p i}=0, \\
& L^{\overline{(i p)}}=G_{n m}^{\overline{(i p)}}+D_{i p}^{n m} \varepsilon_{p}+\sum_{r s}[(r s \mid i p)-(r i \mid p s)] D_{r s}^{n m}-\bar{W}_{i p}=0, \\
& L^{(a p)}=G_{n m}^{(a p)}+D_{p a}^{n m} \varepsilon_{p}-\bar{W}_{p a}=0, \\
& L^{\overline{(a p)}}=G_{n m}^{(a p)}+D_{a p}^{n m} \varepsilon_{p}-\bar{W}_{a p}=0 .
\end{aligned}
$$

Two sets of equations for the $\mathbf{Z}^{n m}$ Lagrange multiplier are obtained by requiring $L^{i a}=L^{\overline{(a i)}}=0$ and $L^{a i}=L^{\overline{(i a)}}=0$, which can be combined into

$$
\Lambda\left|Z^{n m, \mathrm{ov}}, Z^{n m, \mathrm{vo}}\right\rangle-c_{\mathrm{QR}} \Omega_{n m} \Delta\left|X^{n m}, Y^{n m}\right\rangle=-\left|P^{n m}, Q^{n m}\right\rangle .
$$

For derivative couplings based on quadratic response $\left(c_{\mathrm{QR}}=1\right)$, this equation is satisfied by $\left|Z^{\mathrm{ov}}, Z^{\mathrm{vo}}\right\rangle=\left|X^{n m}, Y^{n m}\right\rangle$ such that

$$
\mathbf{D}^{n m, \mathrm{QR}}=\Omega_{n m}{ }^{-1} \gamma^{n m},
$$

while for derivative couplings based on the pseudowavefunction $\left(c_{\mathrm{QR}}=0\right)$,

$$
\left|Z^{n m, \mathrm{PW}}\right\rangle=-\Lambda^{-1}\left|P^{n m}, Q^{n m}\right\rangle .
$$


The Lagrange multiplier $\overline{\mathbf{W}}^{n m}$ is next determined from eqn (38) as

$$
\begin{aligned}
\bar{W}_{i j}^{+n m}= & \frac{1}{2} T_{i j}^{+n m}+\frac{1}{2} H_{i j}^{+}\left[\mathbf{D}^{n m}\right]+\Omega_{n m}{ }^{-1} g_{i j}^{\mathrm{xc}}\left[R^{+n}, R^{+m}\right] \\
& -\frac{\Omega_{n m}{ }^{-1}}{4} \sum_{a} \varepsilon_{a}\left[R_{i a}^{+n} R_{j a}^{+m}+R_{i a}^{-n} R_{j a}^{-m}+(n \leftrightarrow m)\right] \\
& +\bar{\Omega}_{n m} \frac{\Omega_{n m}{ }^{-1}}{4} \sum_{a}\left[R_{i a}^{+n} R_{j a}^{-m}+R_{i a}^{-n} R_{j a}^{+m}+(n \leftrightarrow m)\right],
\end{aligned}
$$

$$
\begin{aligned}
\bar{W}_{a b}^{+n m}= & \frac{1}{2} T_{a b}^{+n m}+\frac{\Omega_{n m}^{-1}}{4} \sum_{i} \varepsilon_{i}\left[R_{i a}^{+n} R_{i b}^{+m}+R_{i a}^{-n} R_{i b}^{-m}+(n \leftrightarrow m)\right] \\
& +\bar{\Omega}_{n m} \frac{\Omega_{n m}^{-1}}{4} \sum_{i}\left[R_{i a}^{+n} R_{i b}^{-m}+R_{i a}^{-n} R_{i b}^{+m}+(n \leftrightarrow m)\right],
\end{aligned}
$$

$$
\begin{aligned}
\bar{W}_{i a}^{+n m}= & c_{\mathrm{QR}} \gamma_{i a}^{n m}+\Omega_{n m}{ }^{-1} \varepsilon_{i} Z_{i a}^{+n m} \\
& +\frac{\Omega_{n m}{ }^{-1}}{4}\left[H_{j i}^{+n} R_{j a}^{+m}+H_{j i}^{-n} R_{j a}^{-m}+(n \leftrightarrow m)\right]
\end{aligned}
$$

$$
\bar{W}_{i j}^{-n m}=\frac{1}{2} T_{i j}^{-n m}+\frac{1}{2} H_{i j}^{-}\left[\mathbf{D}^{n m}\right]
$$$$
-\frac{\Omega_{n m}{ }^{-1}}{4} \sum_{a} \varepsilon_{a}\left[R_{i a}^{+n} R_{j a}^{-m}+R_{i a}^{-n} R_{j a}^{+m}-(n \leftrightarrow m)\right]
$$$$
+\bar{\Omega}_{n m} \frac{\Omega_{n m}{ }^{-1}}{4} \sum_{a}\left[R_{i a}^{+n} R_{j a}^{+m}+R_{i a}^{-n} R_{j a}^{-m}-(n \leftrightarrow m)\right]
$$

$$
\begin{aligned}
\bar{W}_{a b}^{-n m}= & \frac{1}{2} T_{a b}^{-n m}+\frac{1}{2} H_{a b}^{-}\left[\mathbf{D}^{n m}\right] \\
& +\frac{\Omega_{n m}{ }^{-1}}{4} \sum_{i} \varepsilon_{i}\left[R_{i a}^{+m} R_{i b}^{-n}+R_{i a}^{-m} R_{i b}^{+n}-(n \leftrightarrow m)\right] \\
& +\bar{\Omega}_{n m} \frac{\Omega_{n m}{ }^{-1}}{4} \sum_{i}\left[R_{i b}^{+n} R_{i a}^{-m}+R_{i b}^{-n} R_{i a}^{+m}-(n \leftrightarrow m)\right]
\end{aligned}
$$

$$
\begin{aligned}
\bar{W}_{i a}^{-n m}= & c_{\mathrm{QR}} \gamma_{i a}^{n m}+\Omega_{n m}{ }^{-1} \varepsilon_{i} Z_{i a}^{-n m} \\
& +\frac{\Omega_{n m}{ }^{-1}}{4}\left[H_{j i}^{+m} R_{j a}^{-n}+H_{j i}^{-m} R_{j a}^{+n}-(n \leftrightarrow m)\right]
\end{aligned}
$$

Finally, the pair-density in eqn (22) is

$$
\begin{aligned}
\Gamma_{p q r s}^{n m}= & D_{p q}^{0} D_{r s}^{n m}-c_{x} D_{p s}^{0} D_{r q}^{n m} \\
& +\Omega_{n m}{ }^{-1}\left[2 R_{p q}^{+n} R_{r s}^{+m}-c_{x} R_{p r}^{+n} R_{s q}^{+m}-c_{x} R_{p r}^{+n} R_{q s}^{+m}\right. \\
& \left.+c_{x} R_{p r}^{-n} R_{s q}^{-m}-c_{x} R_{p r}^{-n} R_{q s}^{-m}\right]
\end{aligned}
$$

where $\mathbf{R}^{ \pm n}$ should be interpreted as an occupied-virtual-only matrix and $D_{p q}^{(0)}=\delta_{p i} \delta_{q i}$ is the ground state density matrix.

\section{Acknowledgements}

This material is based upon work supported by the U.S. Department of Energy, Office of Basic Energy Sciences, under Award Number DE-SC0018352. S. M. P. is supported by an Arnold O. Beckman Postdoctoral Fellowship. The authors thank Alan Robledo for assistance.

\section{Notes and references}

1 W. Domcke and D. R. Yarkony, Annu. Rev. Phys. Chem., 2012, 63, 325-352.

2 A. Zangwill and P. Soven, Phys. Rev. A: At., Mol., Opt. Phys., 1980, 21, 1561-1572.

3 E. Runge and E. K. U. Gross, Phys. Rev. Lett., 1984, 52, 997-1000.

4 M. Petersilka, U. J. Gossmann and E. K. U. Gross, Phys. Rev. Lett., 1996, 76, 1212-1215.

5 E. K. U. Gross, J. F. Dobson and M. Petersilka, Density Functional Theory II, Springer-Verlag, Berlin/Heidelberg, 1996, pp. 81-172.

6 R. Baer, Chem. Phys. Lett., 2002, 364, 75-79.

7 R. Baer, Y. Kurzweil and L. S. Cederbaum, Isr. J. Chem., 2005, 45, 161-170.

8 J. C. Tully, J. Chem. Phys., 1990, 93, 1061.

9 F. Agostini, B. F. E. Curchod, R. Vuilleumier, I. Tavernelli and E. K. U. Gross, in TDDFT and Quantum-Classical Dynamics: A Universal Tool Describing the Dynamics of Matter, ed. W. Andreoni and S. Yip, Springer International Publishing, Cham, 2018, pp. 1-47.

10 E. Tapavicza, G. D. Bellchambers, J. C. Vincent and F. Furche, Phys. Chem. Chem. Phys., 2013, 15, 18336-18348.

11 C. F. Craig, W. R. Duncan and O. V. Prezhdo, Phys. Rev. Lett., 2005, 95, 163001.

12 E. Tapavicza, I. Tavernelli, U. Rothlisberger, C. Filippi and M. E. Casida, J. Chem. Phys., 2008, 129, 124108.

13 T. Thompson and E. Tapavicza, J. Chem. Phys., 2018, 9, 4758-4764.

14 E. Tapavicza, A. M. Meyer and F. Furche, Phys. Chem. Chem. Phys., 2011, 13, 20986-20998.

15 J. Kim, H. Tao, T. J. Martinez and P. Bucksbaum, J. Phys. B: At., Mol. Opt. Phys., 2015, 48, 164003.

16 O. Schalk, T. Geng, T. Thompson, N. Baluyot, R. D. Thomas, E. Tapavicza and T. Hansson, J. Phys. Chem. A, 2016, 120, 2320-2329.

17 M. Barbatti, J. Pittner, M. Pederzoli, U. Werner, R. Mitrić, V. Bonačić-Koutecký and H. Lischka, Chem. Phys., 2010, 375, 26-34.

18 M. Sapunar, A. Ponzi, S. Chaiwongwattana, M. Malis, A. Prlj, P. Decleva and N. Doslic, Phys. Chem. Chem. Phys., 2015, 17, 19012-19020.

19 M. Wohlgemuth, V. Bonačić-Koutecký and R. Mitrić, J. Chem. Phys., 2011, 135, 054105.

20 L. Du and Z. Lan, J. Chem. Theory Comput., 2015, 11, 1360-1374. 
21 A. V. Akimov, A. J. Neukirch and O. V. Prezhdo, Chem. Rev., 2013, 113, 4496-4565.

22 R. Long, J. Liu and O. V. Prezhdo, J. Am. Chem. Soc., 2016, 138, 3884-3890.

23 W. R. Duncan, W. M. Stier and O. V. Prezhdo, J. Am. Chem. Soc., 2005, 127, 7941-7951.

24 W. R. Duncan, C. F. Craig and O. V. Prezhdo, J. Am. Chem. Soc., 2007, 129, 8528-8543.

25 M. Muuronen, S. M. Parker, E. Berardo, A. Le, M. A. Zwijnenburg and F. Furche, Chem. Sci., 2017, 8, 2179-2183.

26 J. C. Vincent, M. Muuronen, K. C. Pearce, L. N. Mohanam, E. Tapavicza and F. Furche, J. Phys. Chem. Lett., 2016, 7, 4185-4190.

27 Y. Han, B. Rasulev and D. S. Kilin, J. Phys. Chem. Lett., 2017, 8, 3185-3192.

28 E. Tapavicza, I. Tavernelli and U. Rothlisberger, Phys. Rev. Lett., 2007, 98, 023001.

29 M. Wohlgemuth, V. Bonačić-Koutecký and R. Mitrić, J. Chem. Phys., 2010, 133, 194104.

30 I. Tavernelli, B. F. E. Curchod and U. Rothlisberger, Chem. Phys., 2011, 391, 101-109.

31 B. Curchod, T. Penfold, U. Rothlisberger and I. Tavernelli, Cent. Eur. J. Phys., 2013, 11, 1059.

32 T. Nelson, S. Fernandez-Alberti, V. Chernyak, A. E. Roitberg and S. Tretiak, J. Phys. Chem. B, 2011, 115, 5402-5414.

33 Z. Li, B. Suo and W. Liu, J. Chem. Phys., 2014, 141, 244105. 34 Q. Ou, G. D. Bellchambers, F. Furche and J. E. Subotnik, J. Chem. Phys., 2015, 142, 064114.

35 X. Zhang and J. M. Herbert, J. Chem. Phys., 2015, 142, 064109.

36 S. M. Parker, D. Rappoport and F. Furche, J. Chem. Theory Comput., 2018, 14, 807-819.

37 H. Kang, K. T. Lee, B. Jung, Y. J. Ko and S. K. Kim, J. Am. Chem. Soc., 2002, 124, 12958-12959.

38 C. Canuel, M. Mons, F. Piuzzi, B. Tardivel, I. Dimicoli and M. Elhanine, J. Chem. Phys., 2005, 122, 074316.

39 S. Ullrich, T. Schultz, M. Z. Zgierski and A. Stolow, Phys. Chem. Chem. Phys., 2004, 6, 2796.

40 E. Samoylova, H. Lippert, S. Ullrich, I. V. Hertel, W. Radloff and T. Schultz, J. Am. Chem. Soc., 2005, 127, 1782-1786.

41 E. Samoylova, T. Schultz, I. V. Hertel and W. Radloff, Chem. Phys., 2008, 347, 376-382.

42 J. González-Vázquez, L. González, E. Samoylova and T. Schultz, Phys. Chem. Chem. Phys., 2009, 11, 3927.

43 J. J. Szymczak, M. Barbatti, J. T. Soo Hoo, J. A. Adkins, T. L. Windus, D. Nachtigallová and H. Lischka, J. Phys. Chem. A, 2009, 113, 12686-12693.

44 B. K. McFarland, J. P. Farrell, S. Miyabe, F. Tarantelli, A. Aguilar, N. Berrah, C. Bostedt, J. D. Bozek, P. H. Bucksbaum, J. C. Castagna, R. N. Coffee, J. P. Cryan, L. Fang, R. Feifel, K. J. Gaffney, J. M. Glownia, T. J. Martinez, M. Mucke, B. Murphy, A. Natan, T. Osipov, V. S. Petrović, S. Schorb, T. Schultz, L. S. Spector, M. Swiggers, I. Tenney, S. Wang, J. L. White, W. White and M. Gühr, Nat. Commun., 2014, 5, 4235.
45 C. E. Crespo-Hernández, B. Cohen, P. M. Hare and B. Kohler, Chem. Rev., 2004, 104, 1977-2020.

46 S. Perun, A. L. Sobolewski and W. Domcke, J. Phys. Chem. A, 2006, 110, 13238-13244.

47 G. Zechmann and M. Barbatti, J. Phys. Chem. A, 2008, 112, 8273-8279.

48 Z. Lan, E. Fabiano and W. Thiel, J. Phys. Chem. B, 2009, 113, 3548-3555.

49 M. Barbatti, A. J. A. Aquino, J. J. Szymczak, D. Nachtigallová, P. Hobza and H. Lischka, Proc. Natl. Acad. Sci. U. S. A., 2010, 107, 21453-21458.

50 D. Asturiol, B. Lasorne, G. A. Worth, M. A. Robb and L. Blancafort, Phys. Chem. Chem. Phys., 2010, 12, 4949.

51 D. Picconi, V. Barone, A. Lami, F. Santoro and R. Improta, ChemPhysChem, 2011, 12, 1957-1968.

52 L. Stojanović, S. Bai, J. Nagesh, A. Izmaylov, R. CrespoOtero, H. Lischka and M. Barbatti, Molecules, 2016, 21, 1603.

53 R. Improta, F. Santoro and L. Blancafort, Chem. Rev., 2016, 116, 3540-3593.

54 J. C. Tully and R. K. Preston, J. Chem. Phys., 1971, 55, 562-572.

55 N. C. Blais and D. G. Truhlar, J. Chem. Phys., 1983, 79, 1334-1342.

56 M. E. Casida, Time-Dependent Density Functional Response Theory for Molecules, in Recent advances in density functional methods, ed. D. P. Chong, World Scientific, Singapore, 1995, vol. 1, pp. 155-192.

57 F. Furche, J. Chem. Phys., 2001, 114, 5982-5992.

58 E. Gross and W. Kohn, Advances in Quantum Chemistry, in Density Functional Theory of Many-Fermion Systems, ed. P.-O. Löwdin, Academic Press, 1990, vol. 21, pp. 255-291.

59 R. Send and F. Furche, J. Chem. Phys., 2010, 132, 044107.

60 S. Fatehi, E. Alguire, Y. Shao and J. E. Subotnik, J. Chem. Phys., 2011, 135, 234105.

61 X. Zhang and J. M. Herbert, J. Chem. Phys., 2014, 141, 064104.

62 S. M. Parker, S. Roy and F. Furche, J. Chem. Phys., 2016, 145, 134105.

63 F. Furche and R. Ahlrichs, J. Chem. Phys., 2002, 117, 7433-7447.

64 F. Furche, R. Ahlrichs, C. Wachsmann, E. Weber, A. Sobanski, F. Vögtle and S. Grimme, J. Am. Chem. Soc., 2000, 122, 1717-1724.

65 H. Weiss, R. Ahlrichs and M. Häser, J. Chem. Phys., 1993, 99, 1262-1270.

66 O. Treutler and R. Ahlrichs, J. Chem. Phys., 1995, 102, 346-354.

67 R. E. Stratmann, G. E. Scuseria and M. J. Frisch, J. Chem. Phys., 1998, 109, 8218-8224.

68 S. J. A. van Gisbergen, J. G. Snijders and E. J. Baerends, Comput. Phys. Commun., 1999, 118, 119-138.

69 A. Görling, H. H. Heinze, S. P. Ruzankin, M. Staufer and N. Rösch, J. Chem. Phys., 1999, 110, 2785-2799.

70 F. Furche, B. T. Krull, B. D. Nguyen and J. Kwon, J. Chem. Phys., 2016, 144, 174105. 
71 B. I. Dunlap, J. W. D. Connolly and J. R. Sabin, J. Chem. Phys., 1979, 71, 3396-3402.

72 S. Hirata and M. Head-Gordon, Chem. Phys. Lett., 1999, 314, 291-299.

73 S. Yarasi, S. Ng and G. R. Loppnow, J. Phys. Chem. B, 2009, 113, 14336-14342.

74 P. M. Hare, C. E. Crespo-Hernandez and B. Kohler, Proc. Natl. Acad. Sci. U. S. A., 2007, 104, 435-440.

75 D. Asturiol, B. Lasorne, M. A. Robb and L. Blancafort, J. Phys. Chem. A, 2009, 113, 10211-10218.

76 S. Yamazaki and T. Taketsugu, J. Phys. Chem. A, 2011, 116, 491-503.

77 W. Weber and W. Thiel, Theor. Chem. Acc., 2000, 103, 495-506.

78 B. G. Levine, C. Ko, J. Quenneville and T. J. Martnez, Mol. Phys., 2006, 104, 1039-1051.

79 F. Plasser, R. Crespo-Otero, M. Pederzoli, J. Pittner, H. Lischka and M. Barbatti, J. Chem. Theory Comput., 2014, 10, 1395-1405.

80 S. Gozem, F. Melaccio, A. Valentini, M. Filatov, M. HuixRotllant, N. Ferré, L. M. Frutos, C. Angeli, A. I. Krylov, A. A. Granovsky, R. Lindh and M. Olivucci, J. Chem. Theory Comput., 2014, 10, 3074-3084.

81 J. Tao, J. P. Perdew, V. N. Staroverov and G. E. Scuseria, Phys. Rev. Lett., 2003, 91, 146401.

82 S. Grimme, J. Antony, S. Ehrlich and H. Krieg, J. Chem. Phys., 2010, 132, 154104.

83 S. Grimme, S. Ehrlich and L. Goerigk, J. Comput. Chem., 2011, 32, 1456-1465.

84 F. Weigend and R. Ahlrichs, Phys. Chem. Chem. Phys., 2005, 7, 3297-3305.

85 C. Adamo and V. Barone, J. Chem. Phys., 1999, 110, 6158-6170.

86 D. Rappoport and F. Furche, J. Chem. Phys., 2010, 133, 134105.

87 B. Efron, Ann. Stat., 1979, 7, 1-26.

88 S. Nangia, A. W. Jasper, T. F. Miller and D. G. Truhlar, J. Chem. Phys., 2004, 120, 3586-3597.

89 R. Abouaf, J. Pommier and H. Dunet, Chem. Phys. Lett., 2003, 381, 486-494.

90 M. Michaud, M. Bazin and L. O. Sanche, Int. J. Radiat. Biol., 2011, 88, 15-21.

91 I. V. Chernyshova, E. J. Kontros, P. P. Markush and O. B. Shpenik, Opt. Spectrosc., 2013, 115, 645-650.
92 D. Cremer and J. A. Pople, J. Am. Chem. Soc., 1975, 97, 1354-1358.

93 J. E. Subotnik, A. Jain, B. Landry, A. Petit, W. Ouyang and N. Bellonzi, Annu. Rev. Phys. Chem., 2016, 67, 387-417.

94 B. R. Landry, M. J. Falk and J. E. Subotnik, J. Chem. Phys., 2013, 139, 211101.

95 L. Wang, D. Trivedi and O. V. Prezhdo, J. Chem. Theory Comput., 2014, 10, 3598-3605.

96 T. Nelson, S. Fernandez-Alberti, A. E. Roitberg and S. Tretiak, J. Chem. Phys., 2013, 138, 224111.

97 S. M. Parker and F. Furche, in Frontiers of Quantum Chemistry, ed. M. J. Wójcik, H. Nakatsuji, B. Kirtman and Y. Ozaki, Springer Singapore, Singapore, 2018, pp. 69-86.

98 F. Furche, R. Ahlrichs, C. Hättig, W. Klopper, M. Sierka and F. Weigend, Wiley Interdiscip. Rev.: Comput. Mol. Sci., 2013, 4, 91-100.

99 TURBOMOLE V7.4 2019, a development of University of Karlsruhe and Forschungszentrum Karlsruhe $\mathrm{GmbH}$, 1989-2007, TURBOMOLE GmbH, since 2007; available from http://www.turbomole.com.

100 S. M. Parker, S. Roy and F. Furche, Multistate hybrid timedependent density functional theory with surface hopping accurately captures ultrafast thymine photodeactivation, UC Irvine Dash, Dataset, 2019, https://doi.org/10.7280/ D1Q08V.

101 G. Pereira Rodrigues, E. Ventura, S. A. do Monte and M. Barbatti, J. Phys. Chem. A, 2014, 118, 12041-12049.

102 D. Tuna, N. Došlić, M. Mališ, A. L. Sobolewski and W. Domcke, J. Phys. Chem. B, 2015, 119, 2112-2124.

103 D. Fazzi, M. Barbatti and W. Thiel, J. Am. Chem. Soc., 2016, 138, 4502-4511.

104 A. Prlj, N. Došlić and C. Corminboeuf, Phys. Chem. Chem. Phys., 2016, 18, 11606-11609.

105 B. Marchetti and T. N. V. Karsili, Phys. Chem. Chem. Phys., 2016, 18, 3644-3658.

106 M. Mališ and N. Došlić, Molecules, 2017, 22, 493.

107 C. Wiebeler, F. Plasser, G. J. Hedley, A. Ruseckas, I. D. W. Samuel and S. Schumacher, J. Phys. Chem. Lett., 2017, 8, 1086-1092.

108 C. Cisneros, T. Thompson, N. Baluyot, A. C. Smith and E. Tapavicza, Phys. Chem. Chem. Phys., 2017, 19, 5763-5777. 